International Journal of Pure and Applied Mathematics

Volume 93 No. 1 2014, 23-29

ISSN: 1311-8080 (printed version); ISSN: 1314-3395 (on-line version)

url: http://www.ijpam.eu

doi: http://dx.doi.org/10.12732/ijpam.v93i1.3

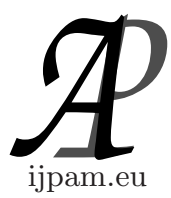

\title{
ON GRADED UNIFORMLY PRIMARY SUBMODULE
}

\author{
Khaldoun Al-Zoubi \\ Department of Mathematics and Statistics \\ Jordan University of Science and Technology \\ P.O. Box 3030, Irbid 22110, JORDAN
}

\begin{abstract}
Let $G$ be a group with identity $e$. Let $R$ be a $G$-graded commutative ring and $M$ a graded $R$-module. In this paper, we introduce the concept of graded uniformly primary submodule and we give a number of results concerning such modules. In fact, our objective is to investigate graded uniformly primary submodules and to examine in particular when graded submodules of a graded module are graded uniformly primary. Special attention has been paid, when graded modules are graded multiplication, to find extra properties of these submodules.
\end{abstract}

AMS Subject Classification: 13A02, 16W50

Key Words: graded primary submodule, graded uniformly primary submodule

\section{Introduction}

Graded primary submodules of graded modules over graded commutative rings have been introduced and studied by various authors, (see, for example [2], [3] ,[5]). Here we introduce the concept of Graded uniformly primary submodules of graded modules over graded commutative rings (see Definition 2) and give a number of its properties.

Before we state some results, let us introduce some notations and terminologies. Let $G$ be a group with identity $e$ and $R$ be a commutative ring.

Received: August 25, 2013

(c) 2014 Academic Publications, Ltd. url: www.acadpubl.eu 
Then $R$ is a $G$-graded ring if there exist additive subgroups $R_{g}$ of $R$ such that $R=\bigoplus_{g \in G} R_{g}$ and $R_{g} R_{h} \subseteq R_{g h}$ for all $g, h \in G$. We denote this by $(R, G)$. The elements of $R_{g}$ are called homogeneous of degree $g$ where $R_{g}$ are additive subgroups of $R$ indexed by the elements $g \in G$. If $x \in R$, then $x$ can be written uniquely as $\sum_{g \in G} x_{g}$, where $x_{g}$ is the component of $x$ in $R_{g}$. Moreover, $h(R)=\bigcup_{g \in G} R_{g}$. Let $I$ be an ideal of $R$. Then $I$ is called graded ideal of $(R, G)$ if $I=\bigoplus_{g \in G}\left(I \cap R_{g}\right)$. Thus, if $x \in I$, then $x=\sum_{g \in G} x_{g}$ with $x_{g} \in I$. An ideal of a $G$-graded ring need not be $G$-graded. For simplicity, we will denote the graded ring $(R, G)$ by $R$. Let $R$ be a $G$-graded ring and $M$ an $R$-module. We say that $M$ is a $G$-graded $R$-module ( or graded $R$-module ) if there exists a family of subgroups $\left\{M_{g}\right\}_{g \in G}$ of $M$ such that $M=\bigoplus_{g \in G} M_{g}$ (as abelian groups ) and $R_{g} M_{h} \subseteq M_{g h}$ for all $g, h \in G$. Here, $R_{g} M_{h}$ denotes the additive subgroup of $M$ consisting of all finite sums of elements $r_{g} s_{h}$ with $r_{g} \in R_{g}$ and $s_{h} \in M_{h}$. Also, we write $h(M)=\cup_{g \in G} M_{g}$ and the elements of $h(M)$ are called homogeneous. Let $M=\underset{g \in G}{\oplus} M_{g}$ be a graded $R$-module and $N$ a submodule of $M$. Then $N$ is called a graded submodule of $M$ if $N=\underset{g \in G}{\oplus} N_{g}$ where $N_{g}=N \cap M_{g}$ for $g \in G$. In this case, $N_{g}$ is called the $g$-component of $N$. For more details, one can look in [4].

Let $R$ be a $G$-graded ring and $M$ a graded $R$-module. The graded radical of a graded ideal $I$, denoted by $\operatorname{Gr}(I)$, is the set of all $x \in R$ such that for each $g \in G$ there exists $n_{g}>0$ with $x_{g}^{n_{g}} \in I$. Note that, if $r$ is a homogeneous element, then $r \in G r(I)$ if and only if $r^{n} \in I$ for some $n \in \mathbb{N}$. A proper graded ideal $P$ of $R$ is said to be graded prime ideal if whenever $r, s \in h(R)$ with $r s \in P$, then either $r \in P$ or $s \in P$. A proper graded ideal $P$ of $R$ is said to be graded primary ideal if whenever $r, s \in h(R)$ with $r s \in P$, then either $r \in P$ or $s \in G r(P)$, For more details, one can look in [6]. A proper graded submodule $N$ of a graded $R$-module $M$ is said to be graded prime submodule if whenever $r \in h(R)$ and $m \in h(M)$ with $r m \in N$, then either $r \in\left(N:_{R} M\right)=\{r \in R: r M \subseteq N\}$ or $m \in N$. A proper graded submodule $N$ of a graded $R$-module $M$ is said to be graded primary submodule if whenever $r \in h(R)$ and $m \in h(M)$ with $r m \in N$, then either $m \in N$ or $r \in G r\left(\left(N:_{R} M\right)\right)$, so $P=G r\left(\left(N:_{R} M\right)\right)$ is a graded prime ideal of $R$, and $N$ is said to be graded $P$-primary submodule. The graded radical of a graded submodule $N$ of a graded $R$-module $M$, denoted by $G r_{M}(N)$, is defined to be the intersection of all graded prime submodules of $M$ containing $N$. If $N$ is not contained in any graded prime submodule of $M$, then $G r_{M}(N)=M$. A graded $R$-module $M$ is said to be graded finitely generated if there exist $x_{g_{1}}, x_{g_{2}}, \ldots, x_{g_{n}} \in h(M)$ such that $M=R x_{g_{1}}+\cdots+R x_{g_{n}}$. For 
more details, one can look in [1], [2], [3], [5].

\section{The Results}

The following Lemma is known, but we write it here for the sake of references.

Lemma 1. Let $R$ be a $G$-graded ring and $M$ a graded $R$-module. Then the following hold:

(i) If $N$ is a graded submodule of $M, r_{g} \in h(R), x_{h} \in h(M)$ and $I$ is a graded ideal of $R$, then $R x_{h}, I N$ and $r_{g} N$ are graded submodules of $M$.

(ii) If $N$ and $K$ are graded submodules of $M$, then $N+K$ is graded submodules of $M$ and $\left(N:_{R} M\right)$ is a graded ideal of $R$.

Definition 2. Let $R$ be a $G$-graded ring and $M$ a graded $R$-module.

(i) A proper graded ideal $Q$ of $R$ is said to be a graded uniformly primary if there exists a positive integer $n$ such that whenever $r_{g}, t_{h} \in h(R)$ with $r_{g} t_{h} \in Q$, then either $r_{g} \in Q$ or $t_{h}^{n} \in Q$. We say that a graded uniformly primary ideal $Q$ has order $s$ (and write $\operatorname{ord}_{R}(Q)=s$ ), if $s$ is the smallest positive integer for which the aforementioned property holds.

(ii) A proper graded submodule $N$ of $M$ is said to be a graded uniformly primary if there exists a positive integer $n$ such that whenever $r_{g} \in h(R)$ and $m_{h} \in h(M)$ with $r_{g} m_{h} \in N$, then either $m_{h} \in N$ or $r_{g}^{n} \in\left(N:_{R} M\right)$. We say that a graded uniformly primary submodule $N$ has order $s$ (and write $\operatorname{ord}_{R}(N)=s$ ), if $s$ is the smallest positive integer for which the aforementioned property holds.

Theorem 3. Let $R$ be a $G$-graded ring, $M$ a graded $R$-module and $N$ a graded submodule of $M$. Then $N$ is a graded uniformly $P$-primary submodule of $M$ if and only if the following two conditions hold:

(i) $N$ is a graded $P$-primary submodule of $M$, and

(ii) there exists a positive integer $n$ such that $P=\left\{r \in R: r_{g}^{n} \in\left(N:_{R} M\right)\right.$ for all $g \in G\}$. Moreover $\operatorname{ord}_{R}(N)=s$ if and only if $s$ is the smallest positive integer for which condition (ii) holds. 
Proof. $(\Rightarrow)$ Let $N$ be a graded uniformly $P$-primary submodule of $M$. Then condition $(i)$ is trivially satisfied. Let $r \in P$ and $g \in G$. Then there are a positive integer $n_{g}$ and $m_{h} \in h(M)$ such that $r_{g}^{n_{g}} m_{h}=r_{g}^{n_{g}-1} r_{g} m_{h} \in N$, but $r_{g}^{n_{g}-1} m_{h} \notin N$. Since $\operatorname{ord}_{R}(N)=s$, we have $r_{g}^{s} \in(N: R M)$, and so condition (ii) is established.

$(\Leftarrow)$ Suppose condition $(i)$ and $(i i)$. Let $r_{g} \in h(R)$ and $m_{h} \in h(M)$ such that $r_{g} m_{h} \in N$ and $m_{h} \notin N$. Since $N$ is a graded $P$-primary, we have $r_{g} \in P$ and condition $(i i)$ provides for positive integer $n$, independent of $r_{g}$, such that $r_{g}^{n} \in\left(N:_{R} M\right)$. Thus $N$ is a graded uniformly primary submodule of $M$. Finally, by the above consideration, $\operatorname{ord}_{R}(N)=s$ if and only if $s$ is the smallest positive integer for which condition $(i i)$ holds.

Theorem 4. Let $R$ be a $G$-graded ring and $Q$ a graded ideal of $R$. Then $Q$ is a graded uniformly $P$-primary ideal of $R$ if and only if the following two conditions hold:

(i) $Q$ is a graded $P$-primary ideal of $R$, and

(ii) There exists a positive integer $n$ such that $P=\left\{r \in R: r_{g}^{n} \in Q\right.$ for all $g \in G\}$. Moreover $\operatorname{ord}_{R}(Q)=s$ if and only if $s$ is the smallest positive integer for which condition (ii) holds.

Proof. The proof is similar to that of Theorem 3.

Theorem 5. Let $R$ be a $G$-graded ring, $M$ a graded $R$-module and $N$ a proper graded submodule of $M$. If there exists a positive integer $s$ such that for any graded ideal $I$ of $R$ and graded submodule $V$ of $M, I V \subseteq N$ implies $V \subseteq N$ or $I^{s} \subseteq\left(N:_{R} M\right)$, then $N$ is a graded uniformly primary submodule with $\operatorname{ord}_{R}(N) \leq s$.

Proof. Let $r_{g} \in h(R)$ and $m_{h} \in h(M)$ such that $r_{g} m_{h} \in N$. So we have $\left(r_{g}\right)\left(m_{h}\right) \subseteq N$ where $\left(r_{g}\right)$ is a graded ideal of $R$ and $\left(m_{h}\right)$ is a graded submodule of $M$. By our assumption we obtain $\left(m_{h}\right) \subseteq N$ or $\left(r_{g}\right)^{s} \subseteq\left(N:_{R} M\right)$ which implies that $m_{h} \in N$ or $r_{g}^{s} \subseteq\left(N:_{R} M\right)$. Thus $N$ is a graded uniformly primary submodule with $\operatorname{ord}_{R}(N) \leq s$.

Lemma 6. Let $R$ be a $G$-graded ring, $M$ a graded $R$-module and $N$ a graded submodule of $M$. If $N$ is a graded uniformly primary submodule of $M$ with order $s$, then for every $m_{\lambda} \in h(M) \backslash N,\left(N:_{R} m_{\lambda}\right)$ is a graded uniformly primary ideal of $R$ and $\operatorname{Gr}\left(\left(N:_{R} m_{\lambda}\right)\right)=\operatorname{Gr}\left(\left(N:_{R} M\right)\right)$. 
Proof. Suppose that $N$ is a graded uniformly primary submodule of $M$ with order $s$. Let $m_{\lambda} \in h(M) \backslash N$ and $r_{g}, t_{h} \in h(R)$ such that $r_{g} t_{h} \in\left(N:_{R} m_{\lambda}\right)$ and $t_{h} \notin\left(N:_{R} m_{\lambda}\right)$. Hence $r_{g} t_{h} m_{\lambda} \in N$ and $t_{h} m_{\lambda} \notin N$. Since $N$ is a graded uniformly primary submodule of $M$ with order $s, r_{g}^{s} \in\left(N:_{R} M\right)$, it follows that $r_{g}^{s} \in\left(N:_{R} m_{\lambda}\right)$. Thus $\left(N:_{R} m_{\lambda}\right)$ is a graded uniformly primary ideal of $R$. It is clear that $\operatorname{Gr}\left(N:_{R} M\right) \subseteq G r\left(\left(N:_{R} m_{\lambda}\right)\right)$. Now we will prove the reverse inclusion. Let $k \in G r\left(\left(N:_{R} m_{\lambda}\right)\right)$ and $g \in G$. Then $k_{g}^{s} m_{\lambda} \in N$. By assumption there exists $l \in \mathbb{Z}^{+}$such that $\left(k_{g}^{s}\right)^{l} \in\left(N:_{R} M\right)$. So $k_{g} \in G r\left(N:_{R} M\right)$. Thus $G r\left(\left(N:_{R} m_{\lambda}\right)\right) \subseteq G r\left(N:_{R} M\right)$.

Theorem 7. Let $R$ be a $G$-graded ring, $M$ a graded finitely generated $R$ module and $N$ a proper graded submodule of $M$. Then the following statements are equivalent.

(i) $N$ is a graded uniformly primary submodule of $M$.

(ii) For $m_{g} \in h(M) \backslash N,\left(N:_{R} m_{g}\right)$ is a graded uniformly primary ideal of $R$ and $\operatorname{Gr}\left(\left(N:_{R} m_{g}\right)\right)=\operatorname{Gr}\left(\left(N:_{R} M\right)\right)$.

Proof. $(i) \Rightarrow$ (ii) See Lemma 6.

$(i i) \Rightarrow(i)$ Since $M$ is a graded finitely generated, there exist $m_{g_{1}}, m_{g_{2}}, \ldots, m_{g_{n}} \in$ $h(M)$ such that $M=R m_{g_{1}}+R m_{g_{2}}+\cdots+R m_{g_{n}}$. Let $s_{i}=\operatorname{ord}_{R}\left(\left(N:_{R}\right.\right.$ $\left.m_{g_{i}}\right)$ ). Without loss of generality, we may assume that $m_{g i} \notin N$. Now, let $r_{g} \in h(R)$ and $m_{h} \in h(M)$ such that $r_{g} m_{h} \in N$ and $m_{h} \notin N$. Hence $r_{g} \in$ $\left(N:_{R} m_{h}\right) \subseteq G r\left(\left(N:_{R} m_{h}\right)\right)=\operatorname{Gr}\left(\left(N:_{R} M\right)\right)=\operatorname{Gr}\left(\left(N:_{R} m_{g_{i}}\right)\right)$. By Theorem $4, r_{g}^{s_{i}} \in\left(N:_{R} m_{g_{i}}\right)$. if $l=\max \left\{\operatorname{ord}_{R}\left(\left(\left(N:_{R} m_{g_{i}}\right)\right): i=1,2, \ldots n\right\}\right.$, then $r_{g}^{l} \in\left(N:_{R} M\right)$. Thus $N$ is a graded uniformly primary submodule of $M$.

Lemma 8. Let $R$ be a $G$-graded ring, $M$ a graded $R$-module and $N$ a graded submodule of $M$. If $N$ is a graded uniformly primary submodule of $M$, then $\left(N:_{R} M\right)$ is a graded uniformly primary ideal of $R$.

Proof. Assume that $N$ is a graded uniformly primary submodule of $M$ of order $s$ and let $r_{g}, t_{h} \in h(R)$ such that $r_{g} t_{h} \in\left(N:_{R} M\right)$ and $r_{g} \notin\left(N:_{R} M\right)$. Then there exists $m_{\lambda} \in h(M) \backslash N$ such that $r_{g} m_{\lambda} \notin N$ and $r_{g} t_{h} m_{\lambda} \in N$. Since $N$ is a graded uniformly primary submodule of $M$ of order $s$, we have $t_{h}^{s} \in\left(N:_{R} M\right)$. Hence $\left(N:_{R} M\right)$ is a graded uniformly primary ideal of $R$.

Recall that a graded $R$-module $M$ is called graded multiplication if for each graded submodule $N$ of $M ; N=I M$ for some graded ideal $I$ of $R$. One can easily show that If $N$ is graded submodule of a graded multiplication module $M$, then $N=\left(N:_{R} M\right) M$, (see, [5, Definition 2]). 
Theorem 9. Let $R$ be a $G$-graded ring, $M$ a graded multiplication $R$ module and $N$ a graded submodule of $M$. Then $N$ is a graded uniformly primary submodule of $M$ if and only if $\left(N:_{R} M\right)$ is a graded uniformly primary ideal of $R$.

Proof. $(\Rightarrow)$ See Lemma 8.

$(\Leftarrow)$ Assume that $(N: R M)$ is a graded uniformly primary ideal of $R$ of order $s$ and let $r_{g} \in h(R)$ and $m_{h} \in h(M)$ such that $r_{g} m_{h} \in N$ and $m_{h} \notin N$. Since $M$ is a graded multiplication, we have $R m_{h}=J M$ for some graded ideal $J$ of $R$. Hence $r_{g} J M \subseteq N$, i.e., $r_{g} J \subseteq\left(N:_{R} M\right)$. Since $\left(N:_{R} M\right)$ is a graded uniformly primary ideal of $R$ of order $s$ and $J \nsubseteq\left(N:_{R} M\right)$, we conclude that $r_{g}^{s} \in\left(N:_{R} M\right)$. Thus $N$ is a graded uniformly primary submodule of $M$.

Lemma 10. [3, Theorem 2.2] Let $R$ be a $G$-graded ring, $M$ a faithful graded multiplication $R$-module and $Q$ a graded primary ideal of $R$. If $r_{g} m_{h} \in$ $Q M$ for $r_{g} \in h(R), m_{h} \in h(M)$, then $r_{g} \in G r(Q)$ or $m_{h} \in Q M$.

Theorem 11. Let $R$ be a $G$-graded ring, $M$ a faithful graded multiplication $R$-module and $Q$ a graded uniformly $P$-primary ideal of $R$ of order $s$ such that $Q M \neq M$. Then $Q M$ is a graded uniformly primary submodule of $M$ of order at most $s$.

Proof. Let $r_{g} \in h(R)$ and $m_{h} \in h(M)$ such that $r_{g} m_{h} \in Q M$ and $m_{h} \notin$ $Q M$. By Lemma 10 and Theorem $4 r_{g} \in G r(Q)=\left\{r \in R: r_{g}^{s} \in Q\right.$ for all $g \in G\}$. Hence $r_{g}^{s} \in Q \subseteq(Q M: R M)$. Therefore $Q M$ is a graded uniformly primary submodule of $M$ of order at most $s$.

\section{Acknowledgments}

The author would like to thanks the referee for his useful comments.

\section{References}

[1] S.E. Atani, On graded prime submodules, Chiang Mai J. Sci., 33, No. 1 (2006), 3-7.

[2] S.E. Atani, F. Farzalipour, On graded secondary modules, Turk. J. Math., 31 (2007), 371-378. 
[3] P. Ghiasvand, F. Farzalipour, On Graded Primary Submodules of Graded Multiplication Modules, Int. J. Alg., 4, No. 9 (2010), 429-434.

[4] C. Nastasescu, F. Van Oystaeyen, Graded Ring Theory, North Holand, Amsterdam, (1982).

[5] K.H. Oral, U. Tekir, A.G. Agargun, On graded prime and primary submodules, Turk. J. Math., 35 (2011), 159-167.

[6] M. Refai, K. Al-Zoubi, On graded primary ideals, Turk. J. Math., 28 (2004), 217-229. 
